\title{
Green Synthesis of Novel GelMA/Carboxymethyl Chitosan Hydrogels and Evaluation of Their Properties for Potential Biomedical Applications
}

\section{Kavoos Razmjooee}

Amirkabir University of Technology Department of Biomedical Engineering

Naghmeh Arabzadeh

Amirkabir University of Technology

\section{Sara Ahmadi}

Amirkabir University of Technology

Azin Rashidy Ahmady

Amirkabir University of Technology Department of Biomedical Engineering

Saeed Saber-Samandari ( $\nabla$ saeedss@aut.ac.ir)

Amirkabir University of Technology

\section{Research Article}

Keywords: GelMA, Carboxymethyl Chitosan (CMC), Hydrogel, Photopolymerization, Acrylamide, Biomaterial.

Posted Date: September 22nd, 2021

DOl: https://doi.org/10.21203/rs.3.rs-904940/v1

License: (c) (i) This work is licensed under a Creative Commons Attribution 4.0 International License. Read Full License 


\section{Abstract}

Gelatin methacrylate (GelMA) hydrogels are the most widely used materials in biomedical applications due to their optimal biological properties and tunable physical characteristics. So far, various studies have been done to improve the properties of GelMA by hybridizing it with chitosan. However, the necessity of acidic solvents usage in these procedures challenges their synthesis process. Herein, we develop an innovative green method to synthesize a novel GelMA hydrogel. In this study, carboxymethyl chitosan $(\mathrm{CMC})$ has been used to modify gelatin to obtain photo-cross-linkable GelMA. The hydrogels with different amounts of $\mathrm{N}, \mathrm{N}$ methylene bisacrylamide as a cross-linking agent were prepared by using the photopolymerization technique, and their chemical and physical properties were characterized. The FTIR spectral analysis and NMR analysis confirmed gelatin and CMC chemical modification and demonstrated GelMA/CMC hydrogel synthesis. Various studies for characterization of the GelMA/CMC hydrogels revealed the tunability of their physical and mechanical properties by changing the cross-linking agent concentration. The synthesized hydrogels exhibited a tailor-made porosity, good mechanical strength, high swelling ratio, and high thermal stability. In addition, the results of L929 fibroblast cell viability assays indicated their excellent biocompatibility. These findings introduce these hydrogels as promising, great candidates to use in various shapes and an extended range of medical applications such as tissue engineering scaffolds, delivery systems, and wound dressings.

\section{Introduction}

Hydrogels are polymeric materials with network linked structure that contains both physical and chemical cross-links. Their particular characteristics are having high water uptake and swelling in aqueous solution [1]. The importance of hydrogels in medical applications was firstly revealed in 1950 [2]. Poly 2-hydroxy ethyl methacrylate (PHEMA) hydrogel was utilized to fabricate contact lenses. This hydrogel exhibited high wettability, elasticity, and biocompatibility. These properties made the PHEMA hydrogel very similar to the natural human body tissues [3]. Afterward, many studies were carried out to develop the synthesis of hydrogels with specified properties, which made them applicable in biomedical researches.

Nowadays, hydrogels have a vast range of applications in the biomedical research area [4]. They have been used to fabricate wound dressings [5], contact lenses [4], membranes [6], drug delivery systems [7], tissue engineering [8], artificial skin [9], biosensors [10],etc. The polymerization methods, choosing monomer, cross-linker, solution, and initiator can play an essential role in the final properties and applications of the synthesized hydrogel [11].

Biocompatibility is a significant feature of hydrogels that depends on surface tension. Surface tension in the intersection of hydrogels and biological tissues is low [12]. Also, due to the high water content, hydrogels' surface is a hydrophilic permeable membrane, which provides biocompatibility $[13,14]$. Some hydrogels' smoothness and elasticity decrease the surface friction of hydrogels with surrounded tissues [15]. Despite all of the hydrogels' unique properties, most of them have shown undesirable mechanical properties that can be a substantial problem, especially after swelling in aqueous solutions [16]. 
For the first time in 2000, Van Den Bulcke and coworkers synthesized a novel methacrylamide-modified gelatin hydrogel by photo-initiated polymerization of gelatin and methacrylic anhydride [17]. This gelatin methacrylamide and also gelatin methacrylate hydrogels were later introduced as GelMA in literature by different authors [18]. GelMA is a hydrogel with a covalently cross-linked network structure that provides desirable physical and chemical properties [19]. Since its unique properties, GelMA has been considered to be used in numerous biomedical applications such as wound dressings [20], Connective tissue [21, 22], Cartilage [23-25], Cardiovascular [26, 27], Bone [28, 29], Tooth Bud [30]. Since then, some researchers have used chitosan to develop the properties of GelMA. For instance, Jang Wook used keratin fiber/chitosan [31]. In the other study, Chen and coworkers synthesized GelMA by using chitosan microspheres and used it to treat osteoarthritis [32]. In addition, Jiali Chen bioprinted multiscale composite scaffolds based on GelMA hydrogel and chitosan microspheres for peripheral nerve tissue engineering [33]. Also, Modaresifar utilized chitosan nanoparticles in the structure of GelMA [34]. These syntheses took place in an acidic environment due to chitosan polymer presence, which eventually limited synthesized GelMA in biomedical applications. Acidic solutions made the GelMA a toxic substrate for living cells and growth factors through the residual acidic reagents trapped in the GelMA structure. So in this study, Carboxymethyl chitosan (CMC) was used. $\mathrm{CMC}$ can dissolve in various $\mathrm{pH}$ environments, and this property is because of carboxymethylation degree. $\mathrm{CMC}$ has many biomedical applications such as tissue engineering, wound healing, tissue engineering and drug/gene delivery bio-imaging [35-37]. In recent years, synthesizing methods based on the green chemistry approaches has become a significant focus of researchers. The essence of green chemistry emphasizes the eliminating the usage of toxic and hazardous solvents and reagents to minimize the environmental impact. Therefore, the replacement of toxic solvents with low or nontoxic solvents is highly recommended [38]. Among the conventional green solvents, including water, n-propyl acetate, i-propyl acetate, 1-butanol, and 2-butanol, water is recognized as the greenest bio-based solvent [39]. Therefore, water-soluble polymers have the potential to be synthesized by green methods.

The most commonly used photoinitiator for GelMA synthesis is 2-hydroxy-1-[4-hydroxyethoxy) phenyl]-2methyl-1-propanone, also known as Irgacure 2959. Heqi et al. evaluated the effect of Irgacure 2959 on cell viability. The results of this study demonstrated the significant cytotoxicity of this photoinitiator at $0,5 \%$ $(w / v)$ concentration and more [40]. Additionally, another problem of most conventional UV photoinitiators such as Irgacure 2959 is insolubility in water due to the presence of the aromatic group in their molecule. This issue makes their usage challenging for water-soluble systems. To solve previously mentioned problems, Ikkai et al. were suggested the usage of ammonium persulfate (APS) as a UV photoinitiator. The results of this study demonstrated that the usage of APS as a UV photoinitiator has several advantages, such as non-toxicity and non-necessity to the use of cross-linkers or pH-adjustment additives [41]. Also, the results of another study, which compared the effectiveness of Irgacure 2959 and APS as initiators, showed the better cytocompatibility and rapid gelation rate for APS compared to Irgacure 2959 [42]. Therefore, in this study, we use ammonium persulfate (APS) as a green and reachable photo initiator instead of complicated and expensive initiator such as Irgacure 2959. 
As we mentioned previously, modification of gelatin hydrogel by methacrylamide, causes optimized characteristics in this hydrogel for biomedical applications. The study performed by Billiet et al. demonstrated that modification of gelatin hydrogel by acrylamide causes better mechanical properties, higher conversion degree, and faster cross-linking kinetics compared to gelatin methacrylamide hydrogel [43]. Therefore, in this study, we suggested using acrylamide for gelatin hydrogel modification to develop a novel GelMA hydrogel.

In this study, we aimed to develop an innovative green method to synthesize a novel GelMA by using Carboxymethyl chitosan (CMC) which is a water soluble polymer [44]. Therefore, the synthesis process was carried out in a completely neutral environment. Furthermore, using $\mathrm{CMC}$ as a part of the hydrogel structure provides unbeatable mechanical properties for the synthesized GelMA. This property makes our novel synthesized GelMA a promising and suitable biomaterial candidate for fabricating sustainable body implants such as artificial vessels, muscles, and ligaments.

\section{Experimental}

\subsection{Materials}

$\mathrm{N}, \mathrm{O}$-Carboxymethyl-chitosan (the degree of substitution is about $80 \%$ and the molecular weight is about 150,000 ) with a $90 \%$ degree of deacetylation and Dialysis membrane with 12-14 kDa cutoff was purchased from Sigma-Aldrich (USA). Acrylamide monomer, Ammonium persulfate (APS, 99\%), Type A porcine skin gelatin, N,Ń-methylenebisacrylamide were obtained from Merck Millipore (Germany).

\subsection{Preparation of hydrogels}

As shown in Table 1. Two solutions were prepared. In the first solution; $0.1 \mathrm{~g}$ gelatin was dissolved in 2.5 $\mathrm{mL}$ Phosphate Buffer Saline (PBS) and stirred for $10 \mathrm{~min}$; then $1 \mathrm{~g}$ acrylamide monomer and different amount of N,N-methylenebisacrylamide that shown in Table 1 as cross-linking agents were added to the solution and stirred roughly for 15 minutes. In the second solution, $0.1 \mathrm{~g}$ Carboxymethyl Chitosan (CMC) was added slowly to the $2.5 \mathrm{~mL}$ PBS solution and heated for $10 \mathrm{~min}$ at $50^{\circ} \mathrm{C}$ to dissolve completely. Finally, both solutions were mixed and stirred for $10 \mathrm{~min}$ at room temperature. $0.1 \mathrm{~g} \mathrm{Ammonium}$ persulfate (APS) as a photoinitiator was added to the final solution. To degassing, the solution was placed in the ultrasonic bath for 15 minutes. The solution was then molded in a cylindrical shape with 16 $\mathrm{mm}$ high and $8 \mathrm{~mm}$ diameter and exposed to UV light (10 W mercury lamp from Philips) at $365 \mathrm{~nm}$ and for 3 minutes to prepare cross-linked hydrogel. For removing unreacted monomer from the structure, the obtained hydrogels were dialyzed against deionized water at $40 \otimes \mathrm{C}$ for seven days, using a dialysis membrane with 12-14 kDa cutoff (D9402, Sigma). Schematic of hydrogel synthesis steps are shown in the graphical abstract (Fig. 1). As shown in Fig. 1, we can synthesize different shapes and dimensions of hydrogels by various molds shapes. 
Table 1

Components of hydrogels in $5 \mathrm{~mL}$ PBS.

\begin{tabular}{|llllll}
\hline Sample & $\begin{array}{l}\text { Acrylamide } \\
(\mathbf{g r})\end{array}$ & $\begin{array}{l}\text { Gelatin } \\
(\mathrm{gr})\end{array}$ & $\begin{array}{l}\text { Carboxymethyl } \\
\text { Chitosan (CMC) } \\
(\mathrm{gr})\end{array}$ & $\begin{array}{l}\text { N,N'- } \\
\text { Methylenebisacrylamide } \\
(\mathrm{gr})\end{array}$ & $\begin{array}{l}\text { Ammonium } \\
\text { persulfate } \\
\text { (APS) } \\
(\mathrm{gr})\end{array}$ \\
\hline $\mathrm{A}$ & 1 & 0.1 & 0.1 & 0.005 & 0.1 \\
\hline B & 1 & 0.1 & 0.1 & 0.025 & 0.1 \\
\hline C & 1 & 0.1 & 0.1 & 0.05 & 0.1 \\
\hline
\end{tabular}

\subsubsection{Fourier transfer infrared spectroscopy (FTIR)}

Chemical analysis of Gelatin, CMC and GelMA were conducted by FTIR spectroscopy over a range of $500-4000 \mathrm{~cm}^{-1}$. FTIR spectra of the hydrogels were operated in the ATR mode by using a Nicolet iS 10 (Thermo Scientific, USA) spectrometer system. The spectrum of samples was recorded with an average scanning rate of $2 \mathrm{~cm}^{-1}$.

\subsubsection{NMR test}

To confirm the synthesis, H NMR spectra of synthesized GelMA (sample B) were prepared using a Bruker Advance 500 spectrometer (Bremen, Germany). The spectra were prepared from the sample at $25^{\circ} \mathrm{C}$ in deuterium oxide $\left(\mathrm{D}_{2} \mathrm{O}\right)$ at $500 \mathrm{MHz}$, and the baseline was corrected before obtaining the integrals of the peaks of interest.

\subsubsection{Scanning electron microscopy (SEM)}

Scanning Electron Microscope (SEM; Seron Technology, South Korea) was utilized to investigate the GelMA hydrogel structure's surface morphology. The samples were first frozen at $-70^{\circ} \mathrm{C}$ and then dried by the freeze-drying method for 24 hours to study SEM's morphology. Before the observation of the sample surfaces, the samples were sputter-coated with a thin layer of gold. Pores' size was measured using SEM micrographs utilizing an image analysis software (ImageJ, National Institute of Health, USA).

\subsubsection{Mechanical properties}

Mechanical properties of GelMA with different ratios of the cross-linking agent were determined by a uniaxial testing machine and compression test (INSTRON 5566, USA) with 10-N load cell under 1 $\mathrm{mm} / \mathrm{min}$ cross-head speed at ambient conditions. All samples for tensile tests were prepared with $3 \times 1 \mathrm{~cm}$ dimensions in a rectangular shape, and for the compression test, all samples in a cylindrical shape with $1.5 \mathrm{~cm}$ high and $1 \mathrm{~cm}$ diameter. To evaluate elasticity, compression tests were performed; the samples were subjected to $50 \%$ strain and five cycles. Ultimate tensile strength, tensile stress-strain curve, tensile modulus, compressive stress-strain curve, compressive stress at $50 \%$ strain, and Compressive modulus 
were obtained from the result of the mechanical test (tensile and compression). All of the mechanical experiment was repeated three times for each sample and were carried out at $25{ }^{\circ} \mathrm{C}$ with an ambient humidity of $28 \%$.

\subsubsection{Thermal Analysis}

To understand the thermal properties of hydrogels, differential scanning calorimetry (DSC) analyses were performed (DSC Diamond series, Perkin Elmer USA), which was equipped with a liquid nitrogen cooling unit. For this purpose, the hydrogel samples with different amounts of cross-linker were heated to temperatures spanning a range of $25-500{ }^{\circ} \mathrm{C}$ in a close aluminum pan at a heating rate of $5{ }^{\circ} \mathrm{C} / \mathrm{min}$. For calibration of the DSC instrument, indium (melting temperature and enthalpy: $T_{m}=156.60^{\circ} \mathrm{C}, \Delta \mathrm{H}_{\mathrm{m}}=28.47$ $\mathrm{J} / \mathrm{g}$ ) was applied as standard. DSC thermograms of samples were recorded and analyzed (Perkin-Elmer Pyris Software) to evaluate peak temperature and area under the thermogram.

\subsubsection{Swelling}

Swelling capacities of the Hydrogels with different amounts of cross-linker were determined by immersing hydrogels in $50 \mathrm{~mL}$ of PBS at $25 \otimes C$ for various times $(1,5,18$, and $24 \mathrm{hr})$. The swelling percentage of the hydrogels were calculated by using the following Equation: $\left(W_{0}\right.$ : Initial weight, $W_{t}$ : the weight of hydrogels in a different time)

Swelling $(\%)=\frac{\mathrm{W}_{\mathrm{t}}-\mathrm{W}_{0}}{W_{0}} \times 100$

\subsubsection{MTT assay}

Cell viability was evaluated using the 3-(4,5-Dimethylthiazol-2-Y1)-2,5-Diphenyltetrazolium Bromide (MTT) assay. L929 fibroblast cell was seeded into 96-well plates in the number of $1 \times 104$ cells per each well and incubated at $37 \otimes \mathrm{C}$ for $24 \mathrm{~h}$. After the incubation, the MTT solution was removed and replaced with $100 \mu \mathrm{l}$ of DMSO. At each time ( 3 and 7 days), the solutions were transferred into a 96-well plate, and the optical density (OD) absorbance was measured at $570 \mathrm{~nm}$ using a TECAN Sunrise microplate reader. The cell viability was measured by the following equation:

Cell viability $(\%)=\frac{\text { Average the optical density of samples }}{\text { Average the optical density of control }} \times 100$

\subsubsection{Statistical Analysis.}

The statistical analyses were carried out by Minitab 17 software (Minitab Inc., State College, USA) using the One-way ANOVA method. All data were expressed as the mean \pm standard deviation (SD). $P<0.05\left(^{*}\right)$ was considered as statistically significant.

\section{Results And Discussion}




\subsection{FIR}

The FTIR spectrum of CMC, gelatin, and photoinitiated radical copolymerized GelMA samples are presented in Fig. 2. A broad peak seen in the $3000-3600 \mathrm{~cm}^{-1}$ area is related to overlapped $\mathrm{O}-\mathrm{H}$ and $\mathrm{N}-\mathrm{H}$ bonds, in CMC and gelatin structure. A similar sharp peak with multi-tips is observed around a wavenumber of $900 \mathrm{~cm}^{-1}$ in both gelatin and GelMA samples. The appearance of this peak in the synthesized GelMA sample spectrum confirms gelatin in the chemical composition. A single peak tip, which is observed in the wavenumber of $1500 \mathrm{~cm}^{-1}$ in the CMC spectrum, is also seen in the GelMA sample spectrum. For all three spectrums, there are some peaks in the area of $1700 \mathrm{~cm}^{-1}$, which is related to the carboxyl functional group.

Also, changes and shifts appeared in peaks of GelMA hydrogel compared to CMC and gelatin, shows that $\mathrm{CMC}$ and gelatin have been chemically modified to GelMA hydrogel.

\subsection{NMR}

To confirm the photo-initiated radical copolymerization of GelMA in this study, the NMR test in the solidstate was performed. As shown in Fig. 3, according to literature review [34], the double tip peak, which is observed approximately in $4.5-5 \mathrm{ppm}$, is related to the presence of chemically modified gelatin with acrylamide the formation of GelMA. This area is indicated by redlined frame a. Grafted methylene functional groups in the synthesized GelMA, created peaks in 1-2.5 ppm area determined by redlined frame $b$. These peaks are due to hydrogens bonded to carbon in methylene functional groups.

\subsection{Investigations of morphological properties}

The microstructure and porosity of all samples were investigated by SEM imaging. As Fig. 4 presents, samples A and B have a porous structure; however, sample $C$ shows no porosity. In sample A which synthesized with a two times lower amount of cross-linker than sample $B$, wide pores on the sample's surface have been created. Although, in sample B, with a higher amount of cross-linker, the porous structure is uniform with narrower pores than sample $A$. Besides, in sample $B$, the porous structure is observed not only on the sample surface layer but also in the hydrogel structure's bulk mass.

\subsection{Mechanical properties}

Mechanical properties of samples A, B, and C were studied by tensile strength and uniaxial compression evaluation. Figure 5a shows the stress-strain curve. As Fig. 5a shows, by increasing cross-linker concentration from samples $A$ to $C$, the tensile modulus enhanced significantly. Tensile modulus is calculated from the slope of the initial linear part of the stress-strain curve, exhibited in Fig. 5b. The tensile modulus of sample $\mathrm{C}$, which contains the highest amount of cross-linker (i.e., $0.05 \mathrm{~g}$ ), is 2.6 -fold and 1.2-fold bigger than sample A and sample B with $0.005 \mathrm{~g}$ and $0.025 \mathrm{~g}$ of the cross-linker, respectively. Figure $5 \mathrm{c}$ presents the strain (\%) of samples at the failure point. As the samples have become stronger from $A$ to $C$, the strain (\%) of them at the rapture point shows lower numbers. 
According to Fig. $5 d$, the ultimate tensile stress of sample B shows 1.2-fold enhancement in comparison with sample $\mathrm{C}$. hence; this result reveals that increasing 5 -fold of cross-linker from sample $A$ to $B$ improves the tensile strength of the copolymerized GelMA and creates a very strong hydrogel structure. However, increasing the amount of cross-linker from $0.025 \mathrm{~g}$ in sample $\mathrm{B}$ to $0.05 \mathrm{~g}$ in sample $\mathrm{C} \nabla$ the ultimate tensile stress decreases dramatically. This result suggests that a large amount of cross-linker prevents the hydrogel formation properly.

Results of compressive stress test presented in Fig. 6a. According to this figure and also Fig. $6 \mathrm{c}$ that shows the stress of samples at $50 \%$ strain, sample $C$ with the highest amount of cross-linker shows 4.2 times higher compressive strength than sample $A$ and 1.7 times higher than sample B. Also, the compressive modulus of the samples (Fig. 6b) follows the same trend in Fig. 6c. Results suggest that the presence of a higher amount of cross-linker leads to the formation of a hydrogel structure capable of resisting higher compression stress.

Fig.7 shows the hysteresis curve between the loading/unloading curves after five cycles of $50 \%$ strain. All three samples have low hysteresis and have regained their original shape after five cycles of compression loading. Still, specimens $A$ and $B$ have more negligible hysteresis than specimen $C$, which can be due to less cross-linker in the structure of specimens $A$ and $B$. Therefore, it can be concluded that by increasing the amount of cross-linker from $0.025 \mathrm{~g}$ in sample $B$ to $0.05 \mathrm{~g}$ in sample $C$, the structure becomes denser and has less elasticity, and causes increased hysteresis.

\subsection{Thermal Analysis}

DSC analysis was applied to investigate the effect of the ratios of the cross-linking agent on the thermal behavior of hydrogels. As shown in Fig. 8, samples $A, B$, and $C$ displayed endothermic peaks at $65^{\circ} \mathrm{C}$, $74^{\circ} \mathrm{C}$, and $90^{\circ} \mathrm{C}$, respectively. As suggested in the literature, these endothermic peaks are attributed to the glass transition of amino acid blocks in the peptide chain of gelatin [45].

A series of board multiple endothermic peaks were observed for all samples at around $145-205^{\circ} \mathrm{C}$, corresponding to the transition from the crystalline state to the amorphous state. According to Fig. 8 , the area under the thermogram curves reduces from sample $A$ to $C$. This reduction in the area under the thermograms indicates decreasing in energy consumption from samples $A$ to $C$, which shows the amorphous structure of sample $\mathrm{C}$. This issue can be explained by the fact that the amount of cross-linker in sample $C$ is several times higher than samples $A$ and $B$ (10 and 5 times, respectively). In other words, the increase in cross-link density of sample $\mathrm{C}$ has reduced its crystallinity and significantly reduced the area under the curve in the transition zone.

Based on the literature [46], the exothermic transitions, which observed at around $220^{\circ} \mathrm{C}, 225^{\circ} \mathrm{C}$, and $235^{\circ} \mathrm{C}$ for samples $A, B$, and $C$, respectively, are attributed to their $C M C$ thermal decompositions. Sharp endothermic peaks observed at 280,302 , and $304^{\circ} \mathrm{C}$ for samples $\mathrm{A}, \mathrm{B}$, and $\mathrm{C}$, respectively, are attributed to the thermal degradation of gelatin contents of the hydrogels. These results indicate the higher thermal stability of sample $\mathrm{C}$ than other samples. 


\subsection{Swelling}

The wettability of GelMA samples plays a vital role in the possibility of cell growth and nutrition in their future biomedical applications, such as body part implants. In addition, the structural stability of synthesized GelMA hydrogel in an aqueous environment needs to be investigated. Therefore, the swelling behavior of samples was studied in PBS solution (Fig. 9). The resulting sample A with a minimum amount of cross-linker shows the highest swelling percentage (i.e., 375\%), followed by sample B with $100 \%$ and sample $C$ with a $70 \%$ swelling ratio during 24 hours. If the samples are considered to be utilized as wound dressing pads, the highest swelling of sample A means the maximum water uptake from the environment is desirable. The swelling results of samples $B$ and $C$ are not significantly different from each other $(P>0.05)$, but sample $A$ is very different from samples $B$ and $C(P<0.05)$.

The swelling behavior of the hydrogel is also an indicator of the hydrogel's cross-link density [25]. The results indicated that the swelling percentages of the samples decreased by enhancing the amount of cross-linker, which could be ascribed to the fact that the cross-linking density of the samples is increased.

\subsection{MTT}

As a matter of the fact that the photo-initiated radical copolymerized GelMA is considered to be used in possible biomedical applications, the cytotoxicity of samples was investigated. Hence, the viability of L929 cells of sample extracts was evaluated by applying an MTT assay after 3 and 7 days. According to Fig. 10, the cell viability of all samples after both 3 and 7 days is higher than $95 \%$. The cytotoxicity of samples shows very desirable results in comparison with the control sample. Therefore, the synthesized GelMA exhibits no toxicity and possesses a high proliferation rate, making it suitable for further biomedical application.

\section{Conclusion}

We have successfully synthesized novel biocompatible GelMA/CMC hydrogels with green solvents and UV photo-crosslinking in this study. These hydrogels were synthesized with three different amounts of cross-linker $(0.005 \mathrm{~g}, 0.025 \mathrm{~g}$, and $0.05 \mathrm{~g})$, and their properties of surface morphology, mechanical properties, thermal characteristics, swelling capacities, and cell viability of these samples were systematically compared. The pore size of hydrogel samples decreases significantly with an increase in the cross-linker concentration. The results obtained from the mechanical analysis revealed that by the increase in the cross-linker concentration of samples, the tensile modulus and tensile stress would be enhanced. Also, characterization analysis results indicated that an increase in the amount of cross-linker increases the hysteresis and thermal stability of samples. The swelling results of samples showed that an increase in the cross-linker concentration causes a reduction of swelling percentage. Additionally, cell viability assay exhibited no toxicity for synthesized hydrogel samples and indicated their excellent cell compatibility properties. 
The results of this research demonstrated that the hydrogel properties are tunable by the control of crosslinker concentration. The capability to adjust the properties of this novel GelMA hydrogel to the optimum level for each specific application and their ability to photopatterned polymerization, by which they can form various shapes and dimensions, allow it to be used in an extensive range of medical applications. Therefore, these hydrogels have promising potential to be used in multiple tissue engineering scaffolds such as vascular, bone, cartilage, nerve, and skin tissue engineering.

\section{Declarations}

Acknowledgements and funding sources: This research did not receive any specific grant from funding agencies in the public, commercial, or not-for-profit sectors.

\section{References}

1. Ahmed EM (2015) Hydrogel: Preparation, characterization, and applications: A review. J Adv Res 6:105-121

2. Kirschner CM, Anseth KS (2013) Hydrogels in healthcare: from static to dynamic material microenvironments. Acta Mater 61:931-944

3. Wichterle O, Lim D (1960) Hydrophilic gels for biological use. Nature 185:117-118

4. Alvarez-Lorenzo C, Anguiano-Igea S, Varela-García A, et al (2019) Bioinspired hydrogels for drugeluting contact lenses. Acta Biomater 84:49-62

5. MÂNDRU M, SPIRIDON M, AFLORI M, et al (2019) HYDROGEL-ACTIVE WOUND DRESSING FOR DRUG DELIVERY IN WOUND HEALING AND SKIN REGENERATION. Int J Med Dent 23:

6. Decock J, Schlenk M, Salmon J-B (2018) In situ photo-patterning of pressure-resistant hydrogel membranes with controlled permeabilities in PEGDA microfluidic channels. Lab Chip 18:1075-1083

7. Bagherifard S, Tamayol A, Mostafalu P, et al (2016) Dermal patch with integrated flexible heater for on demand drug delivery. Adv Healthc Mater 5:175-184

8. Fares MM, Sani ES, Lara RP, et al (2018) Interpenetrating network gelatin methacryloyl (GelMA) and pectin-g-PCL hydrogels with tunable properties for tissue engineering. Biomater Sci 6:2938-2950

9. Uehara M, Li X, Sheikhi A, et al (2019) Anti-IL-6 eluting immunomodulatory biomaterials prolong skin allograft survival. Sci Rep 9:1-13

10. Walker BW, Lara RP, Mogadam E, et al (2019) Rational design of microfabricated electroconductive hydrogels for biomedical applications. Prog Polym Sci 92:135-157

11. Zhao X, Lang Q, Yildirimer $L$, et al (2016) Photocrosslinkable gelatin hydrogel for epidermal tissue engineering. Adv Healthc Mater 5:108-118

12. Zimberlin JA (2009) Mechanics of hydrogels and biological tissues

13. Li J, Mooney DJ (2016) Designing hydrogels for controlled drug delivery. Nat Rev Mater 1:1-17 
14. Caló E, Khutoryanskiy V V (2015) Biomedical applications of hydrogels: A review of patents and commercial products. Eur Polym J 65:252-267

15. Mantha S, Pillai S, Khayambashi P, et al (2019) Smart hydrogels in tissue engineering and regenerative medicine. Materials (Basel) 12:3323

16. Hua J, Ng PF, Fei B (2018) High-strength hydrogels: Microstructure design, characterization and applications. J Polym Sci Part B Polym Phys 56:1325-1335

17. Van Den Bulcke Al, Bogdanov B, De Rooze N, et al (2000) Structural and rheological properties of methacrylamide modified gelatin hydrogels. Biomacromolecules 1:31-38

18. Yue K, Trujillo-de Santiago G, Alvarez MM, et al (2015) Synthesis, properties, and biomedical applications of gelatin methacryloyl (GelMA) hydrogels. Biomaterials 73:254-271

19. Young AT, White OC, Daniele MA (2020) Rheological Properties of Coordinated Physical Gelation and Chemical Crosslinking in Gelatin Methacryloyl (GelMA) Hydrogels. Macromol Biosci 20:2000183

20. Annabi N, Rana D, Sani ES, et al (2017) Engineering a sprayable and elastic hydrogel adhesive with antimicrobial properties for wound healing. Biomaterials 139:229-243

21. Cha C, Shin SR, Gao X, et al (2014) Controlling mechanical properties of cell-laden hydrogels by covalent incorporation of graphene oxide. Small 10:514-523

22. Wang H, Zhou L, Liao J, et al (2014) Cell-laden photocrosslinked GelMA-DexMA copolymer hydrogels with tunable mechanical properties for tissue engineering. J Mater Sci Mater Med 25:2173-2183

23. Shin H, Olsen BD, Khademhosseini A (2012) The mechanical properties and cytotoxicity of cell-laden double-network hydrogels based on photocrosslinkable gelatin and gellan gum biomacromolecules. Biomaterials 33:3143-3152

24. Levett PA, Melchels FPW, Schrobback K, et al (2014) A biomimetic extracellular matrix for cartilage tissue engineering centered on photocurable gelatin, hyaluronic acid and chondroitin sulfate. Acta Biomater 10:214-223

25. Gan D, Xu T, Xing W, et al (2019) Mussel-inspired dopamine oligomer intercalated tough and resilient gelatin methacryloyl (GelMA) hydrogels for cartilage regeneration. J Mater Chem B 7:1716-1725

26. Camci-Unal G, Cuttica D, Annabi N, et al (2013) Synthesis and characterization of hybrid hyaluronic acid-gelatin hydrogels. Biomacromolecules 14:1085-1092

27. Qi H, Du Y, Wang L, et al (2010) Patterned differentiation of individual embryoid bodies in spatially organized 3D hybrid microgels. Adv Mater 22:5276-5281

28. Fathi A, Lee S, Breen A, et al (2014) Enhancing the mechanical properties and physical stability of biomimetic polymer hydrogels for micro-patterning and tissue engineering applications. Eur Polym $\mathrm{J}$ 59:161-170

29. Kang H, Shih Y-R V, Hwang Y, et al (2014) Mineralized gelatin methacrylate-based matrices induce osteogenic differentiation of human induced pluripotent stem cells. Acta Biomater 10:4961-4970 
30. Smith EE, Yelick PC (2019) Bioengineering tooth bud constructs using GelMA hydrogel. In: Odontogenesis. Springer, pp 139-150

31. Kim JW, Kim MJ, Ki CS, et al (2017) Fabrication of bi-layer scaffold of keratin nanofiber and gelatinmethacrylate hydrogel: Implications for skin graft. Int J Biol Macromol 105:541-548

32. Chen P, Xia C, Mei S, et al (2016) Intra-articular delivery of sinomenium encapsulated by chitosan microspheres and photo-crosslinked GelMA hydrogel ameliorates osteoarthritis by effectively regulating autophagy. Biomaterials $81: 1-13$

33. Chen J, Huang D, Wang L, et al (2020) 3D bioprinted multiscale composite scaffolds based on gelatin methacryloyl (GelMA)/chitosan microspheres as a modular bioink for enhancing 3D neurite outgrowth and elongation. J Colloid Interface Sci 574:162-173

34. Modaresifar K, Hadjizadeh A, Niknejad H (2018) Design and fabrication of GelMA/chitosan nanoparticles composite hydrogel for angiogenic growth factor delivery. Artif cells, nanomedicine, Biotechnol 46:1799-1808

35. Shariatinia Z (2018) Carboxymethyl chitosan: Properties and biomedical applications. Int J Biol Macromol 120:1406-1419

36. Fazli Y, Shariatinia Z (2017) Controlled release of cefazolin sodium antibiotic drug from electrospun chitosan-polyethylene oxide nanofibrous Mats. Mater Sci Eng C 71:641-652

37. Anitha A, Maya S, Deepa N, et al (2011) Efficient water soluble O-carboxymethyl chitosan nanocarrier for the delivery of curcumin to cancer cells. Carbohydr Polym 83:452-461

38. Sheldon RA (2018) Metrics of green chemistry and sustainability: past, present, and future. ACS Sustain Chem Eng 6:32-48

39. Byrne FP, Jin S, Paggiola G, et al (2016) Tools and techniques for solvent selection: green solvent selection guides. Sustain Chem Process 4:1-24

40. Xu H, Casillas J, Krishnamoorthy S, Xu C (2020) Effects of Irgacure 2959 and lithium phenyl-2, 4, 6trimethylbenzoylphosphinate on cell viability, physical properties, and microstructure in 3D bioprinting of vascular-like constructs. Biomed Mater 15:55021

41. Ikkai F, Adachi E (2004) Novel method of producing polymer gels in aqueous solution using UV irradiation. Macromol Rapid Commun 25:1514-1517

42. Wilems TS, Lu X, Kurosu YE, et al (2017) Effects of free radical initiators on polyethylene glycol dimethacrylate hydrogel properties and biocompatibility. J Biomed Mater Res Part A 105:3059-3068

43. Billiet T, Gasse B Van, Gevaert E, et al (2013) Quantitative Contrasts in the Photopolymerization of Acrylamide and Methacrylamide-F unctionalized Gelatin Hydrogel Building Blocks. Macromol Biosci 13:1531-1545

44. Che Nan NF, Zainuddin N, Ahmad M (2019) Preparation and Swelling Study of CMC Hydrogel as Potential Superabsorbent. Pertanika J Sci Technol 27:

45. Aldana AA, Malatto L, Rehman MAU, et al (2019) Fabrication of gelatin methacrylate (GelMA) scaffolds with nano-and micro-topographical and morphological features. Nanomaterials 9:120 
46. Vaghani SS, Patel MM, Satish CS, et al (2012) Synthesis and characterization of carboxymethyl chitosan hydrogel: Application as site specific delivery for lercanidipine hydrochloride. Bull Mater Sci 35:1133-1142

\section{Figures}

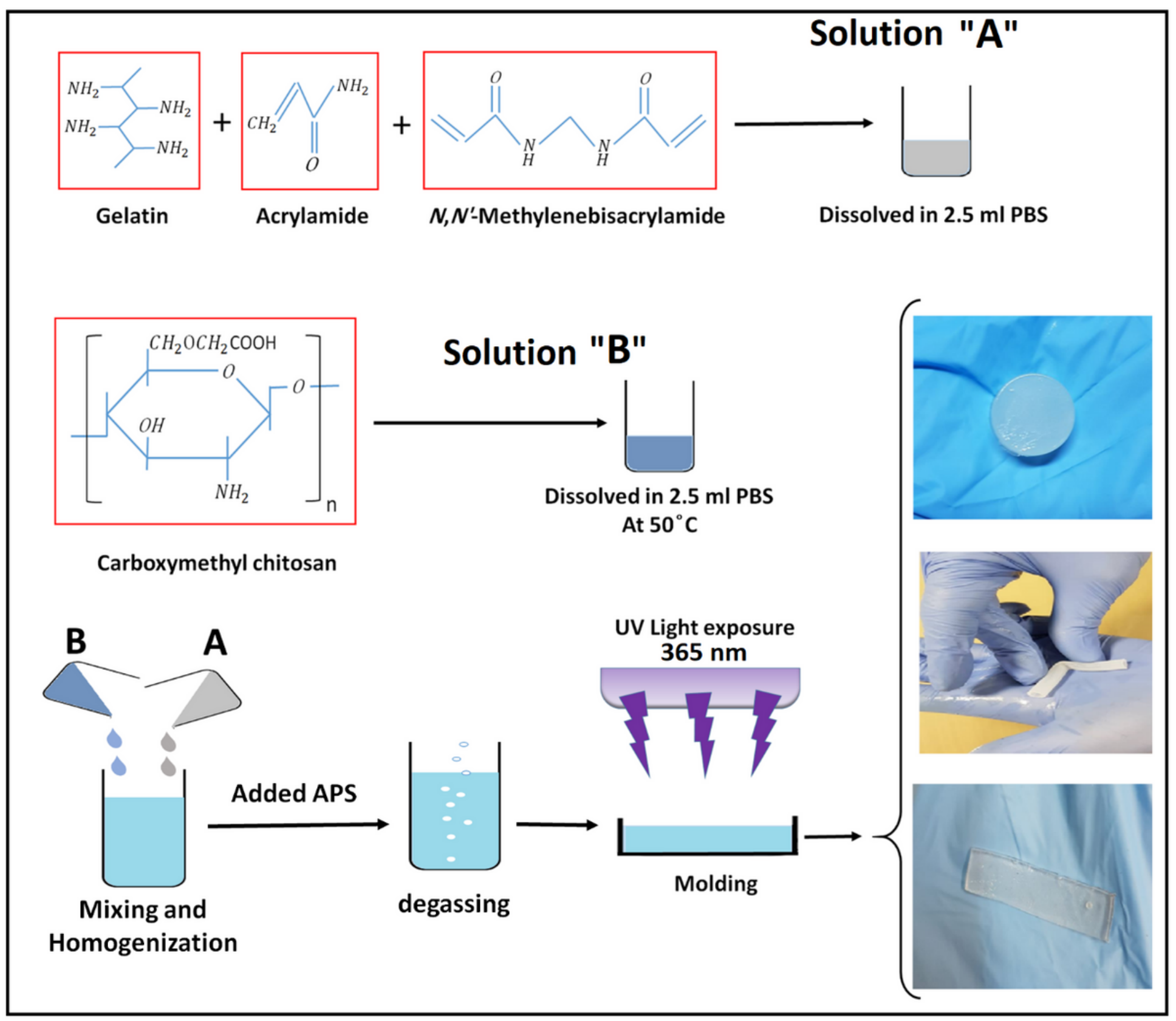

Figure 1

Schematic of hydrogel synthesis. 


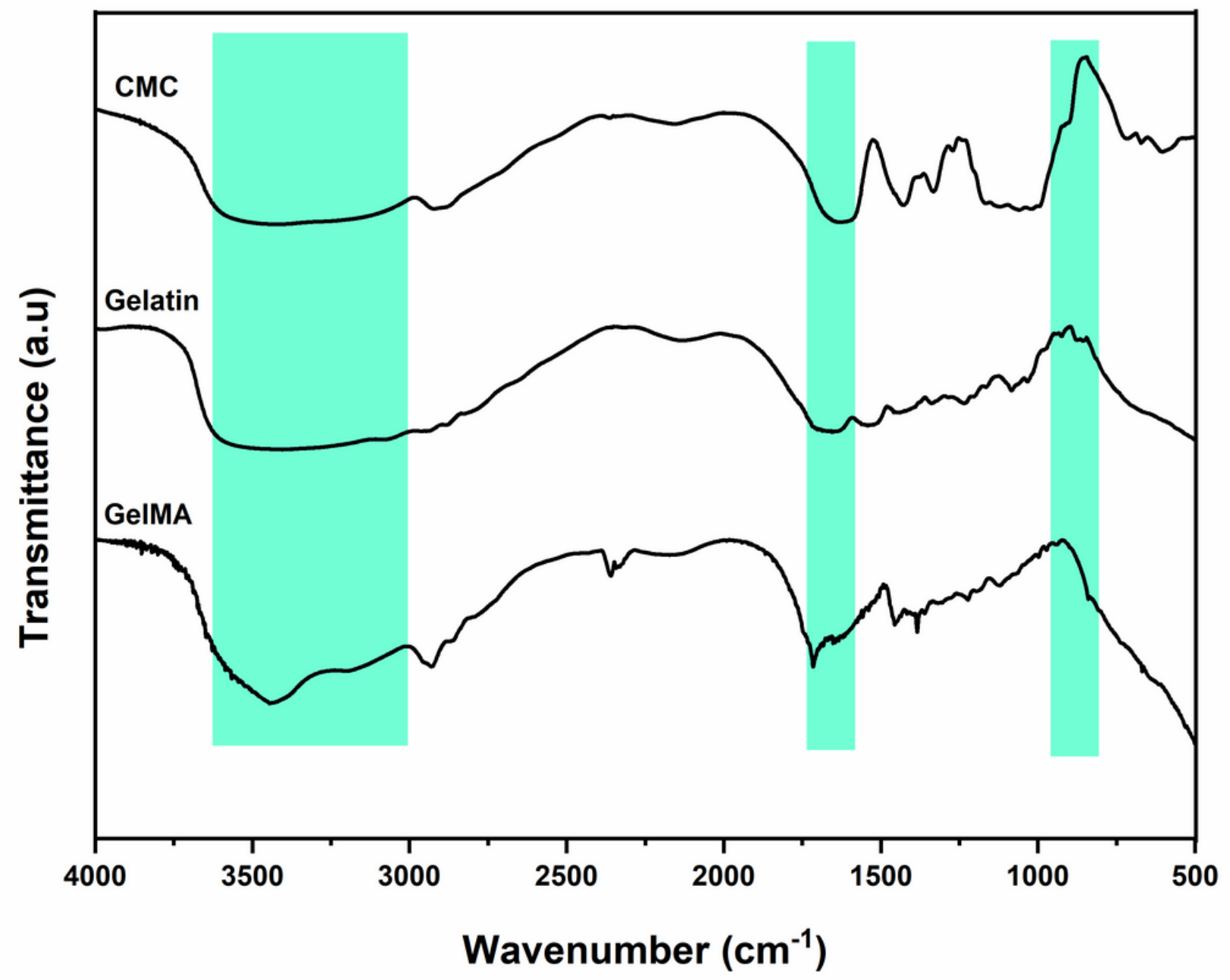

Figure 2

ATR-FTIR spectrum of CMC, Gelatin and GelMA hydrogel with $0.0 .25 \mathrm{~g}$ cross-linker.

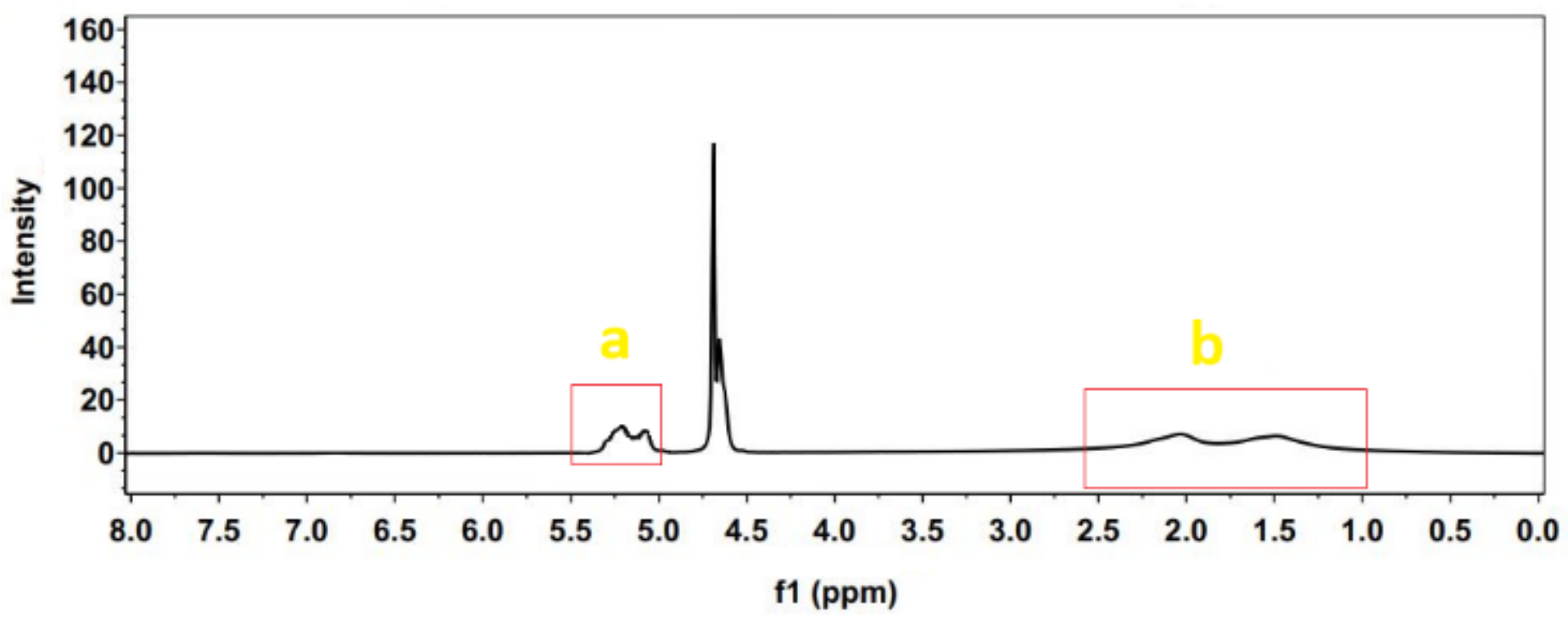

Figure 3 
NMR of GelMA hydrogel with $0.0 .25 \mathrm{~g}$ cross-linker.
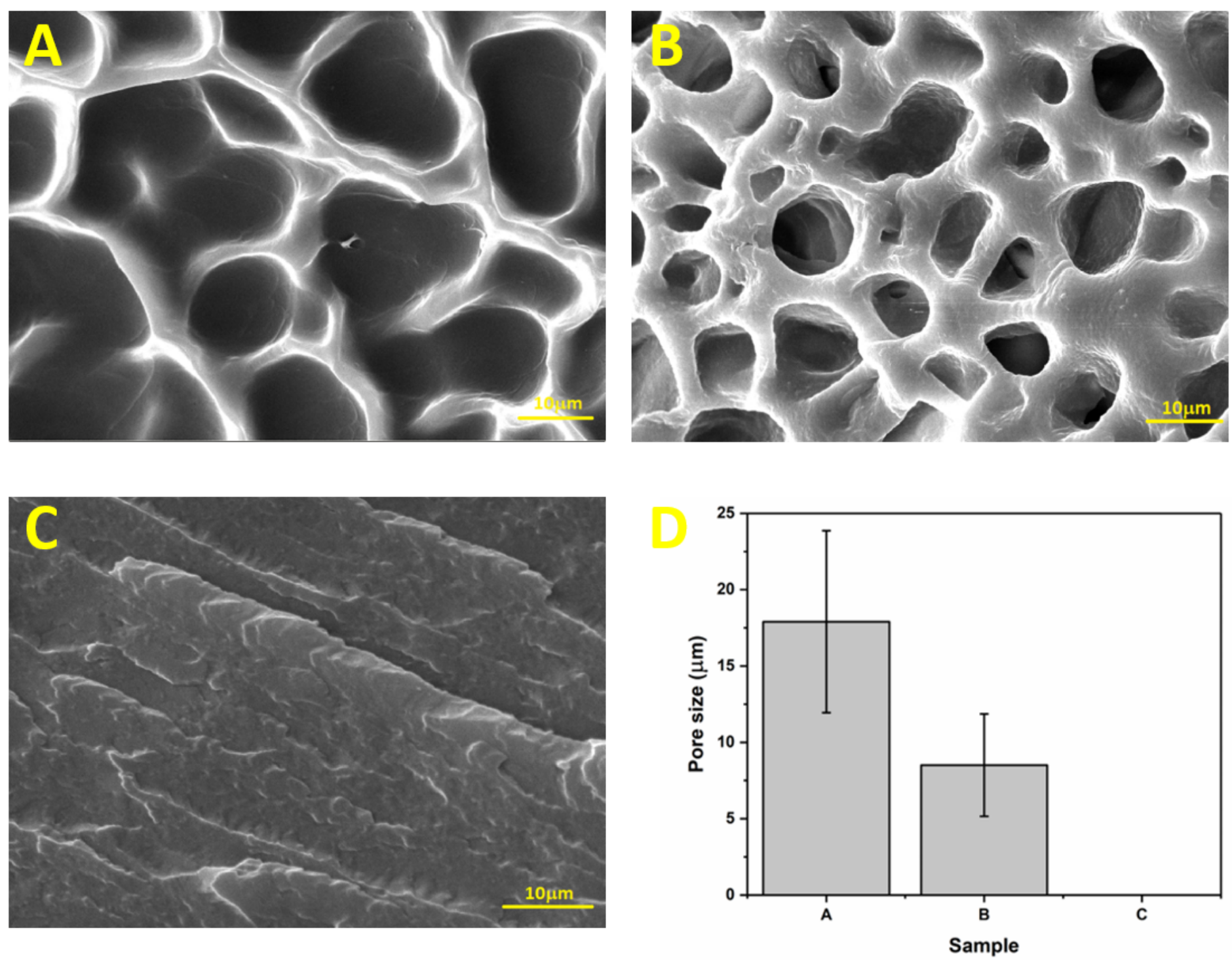

Figure 4

SEM images of GelMA hydrogels with different amounts of cross-linker. Sample A $(0.005 \mathrm{~g})$, Sample B (0.025g), Sample C (0.05g). 

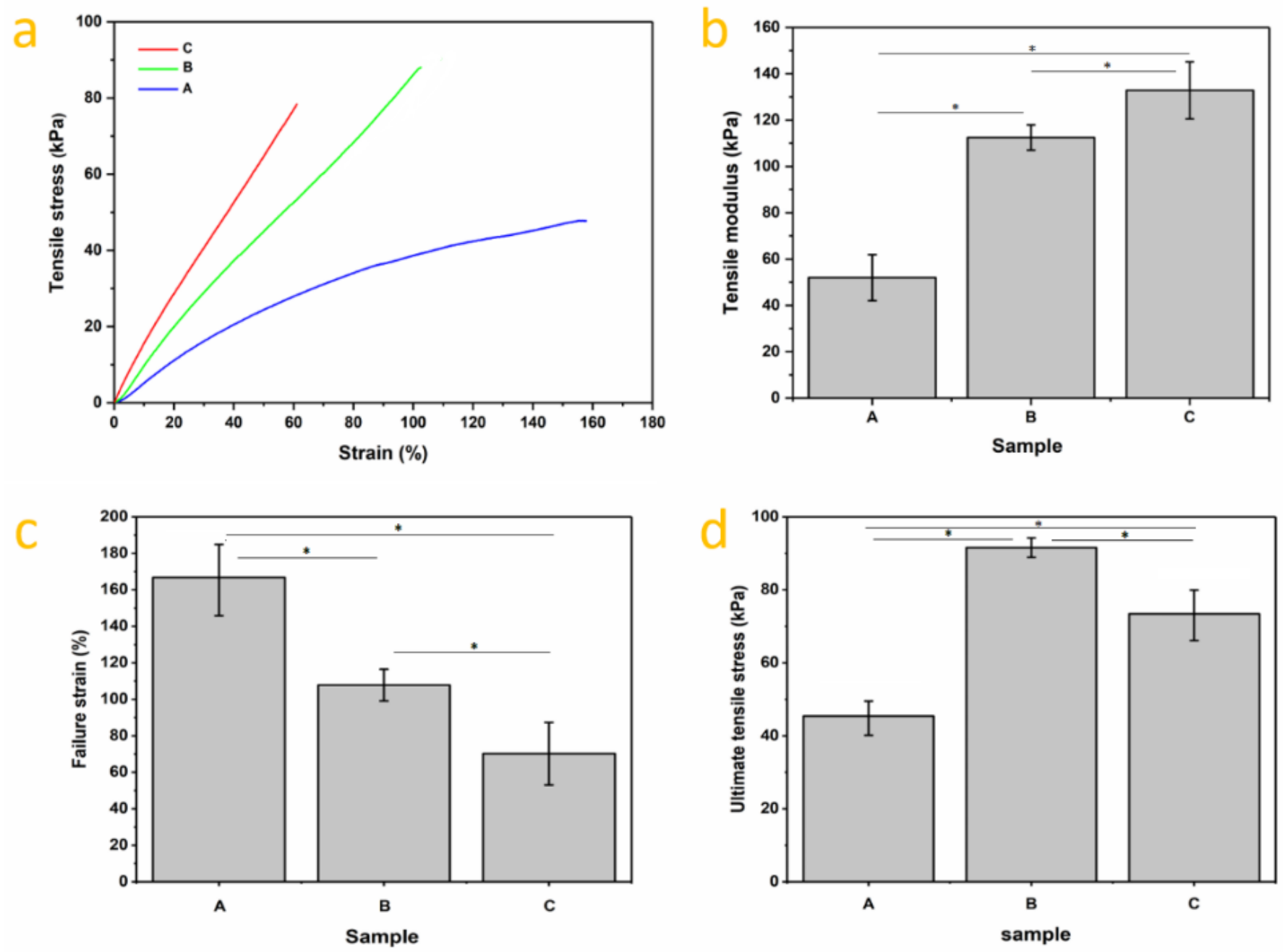

Figure 5

Tensile stress-strain curve (a), tensile modulus (b), strain (\%) of samples at failure point (c), ultimate tensile stress $(d),(* P<0.05)$. 

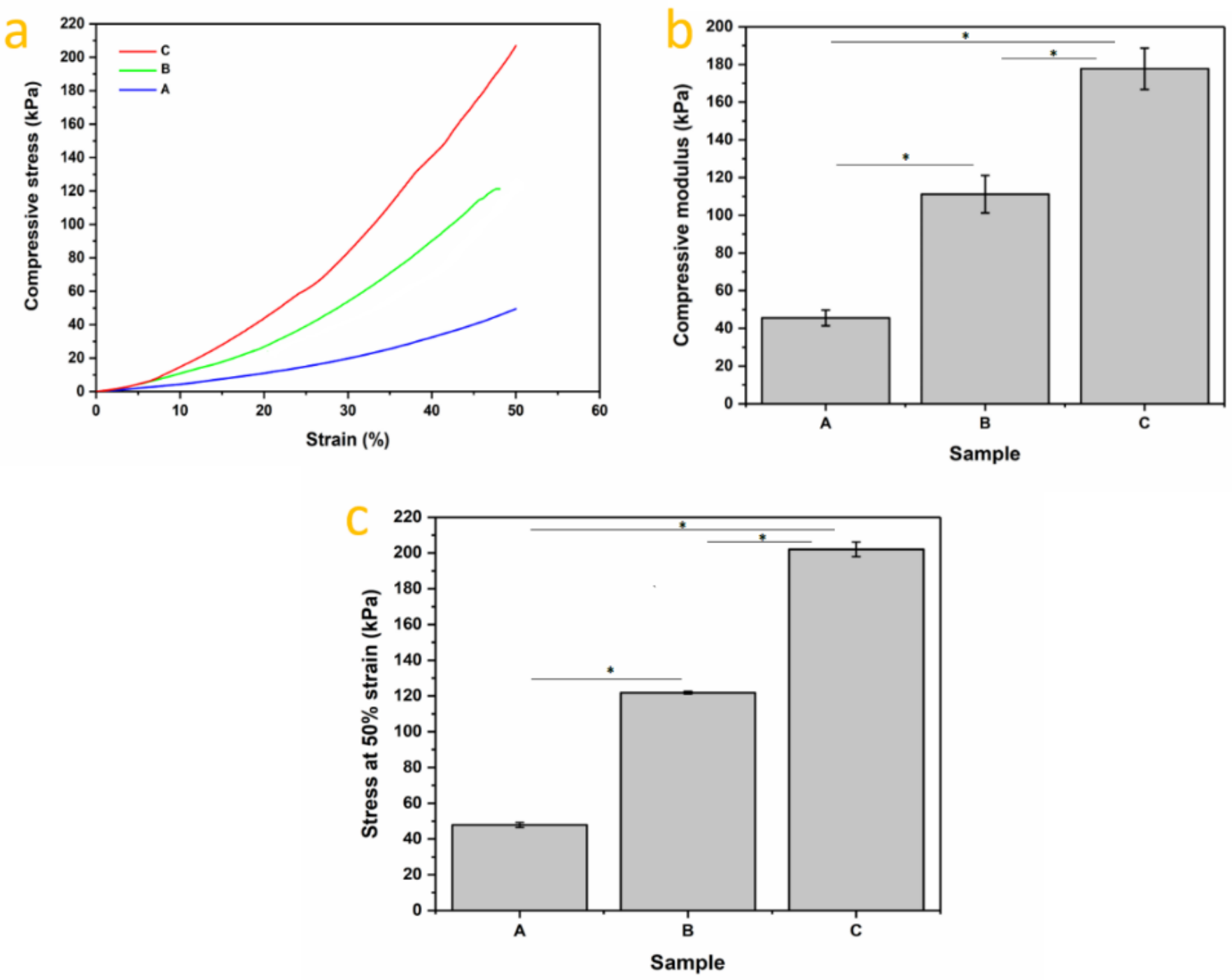

Figure 6

Compressive stress-strain curve (a), Compressive modulus (b), stress of samples at $50 \%$ strain (c), ( $\mathrm{P}<$ 0.05). 

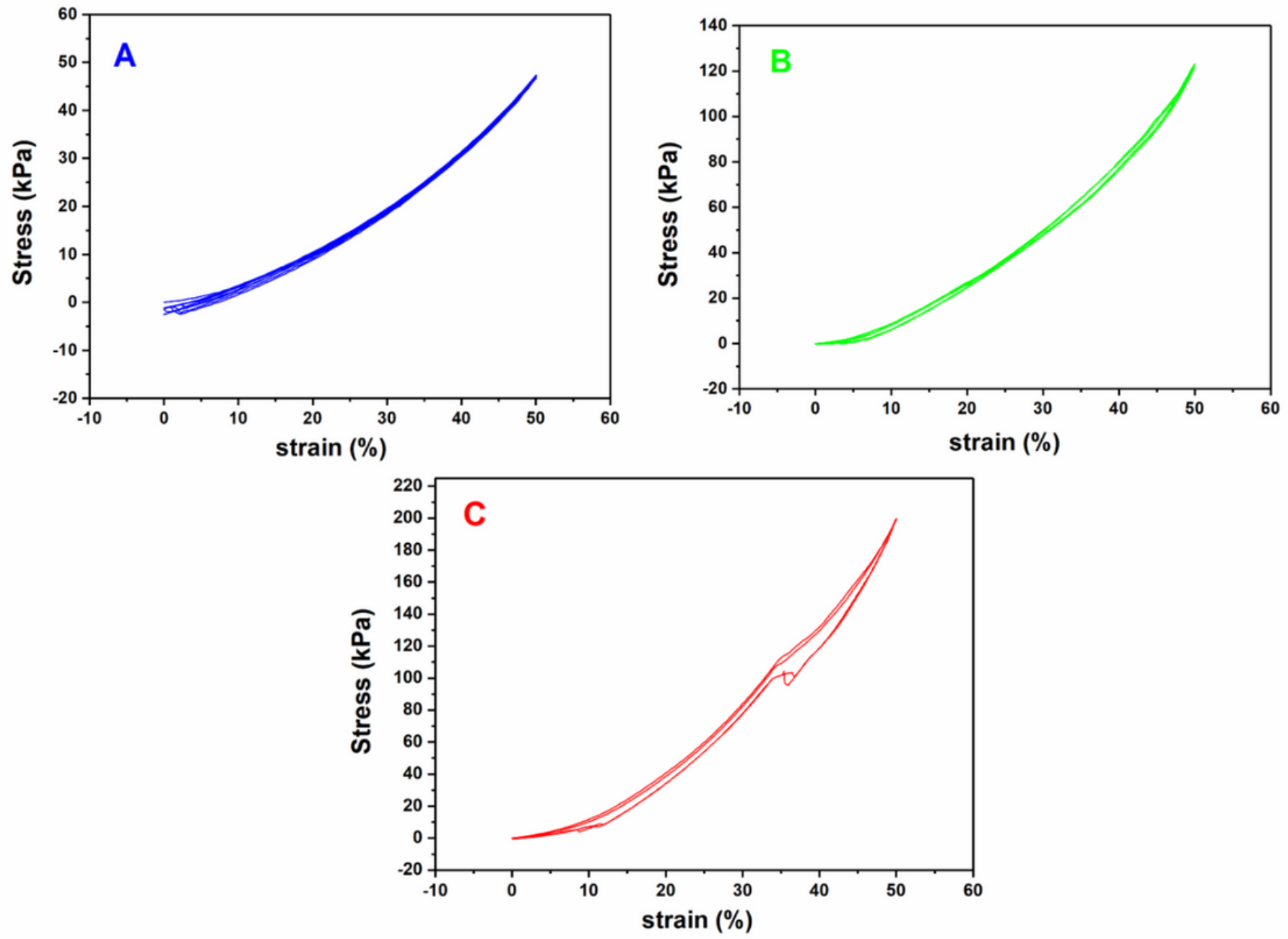

\section{Figure 7}

Hysteresis curve between the loading/unloading curves after 5 cycles of $50 \%$ strain for GelMA hydrogels with different amount of cross-linker. 


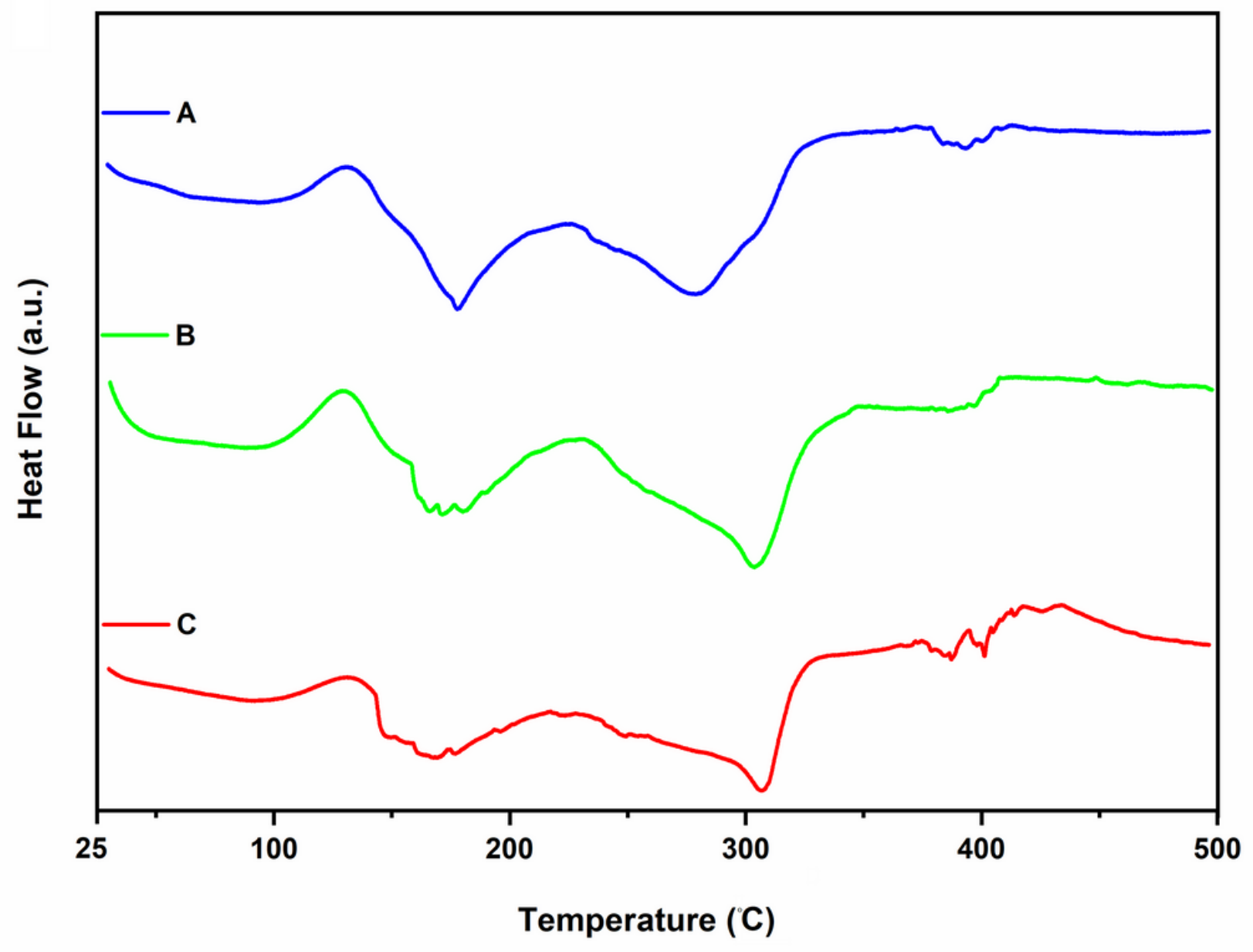

Figure 8

DSC thermogram of GelMA hydrogels with different amounts of cross-linker, Sample A $(0.005 \mathrm{~g})$, Sample B (0.025g), Sample C (0.05g). 


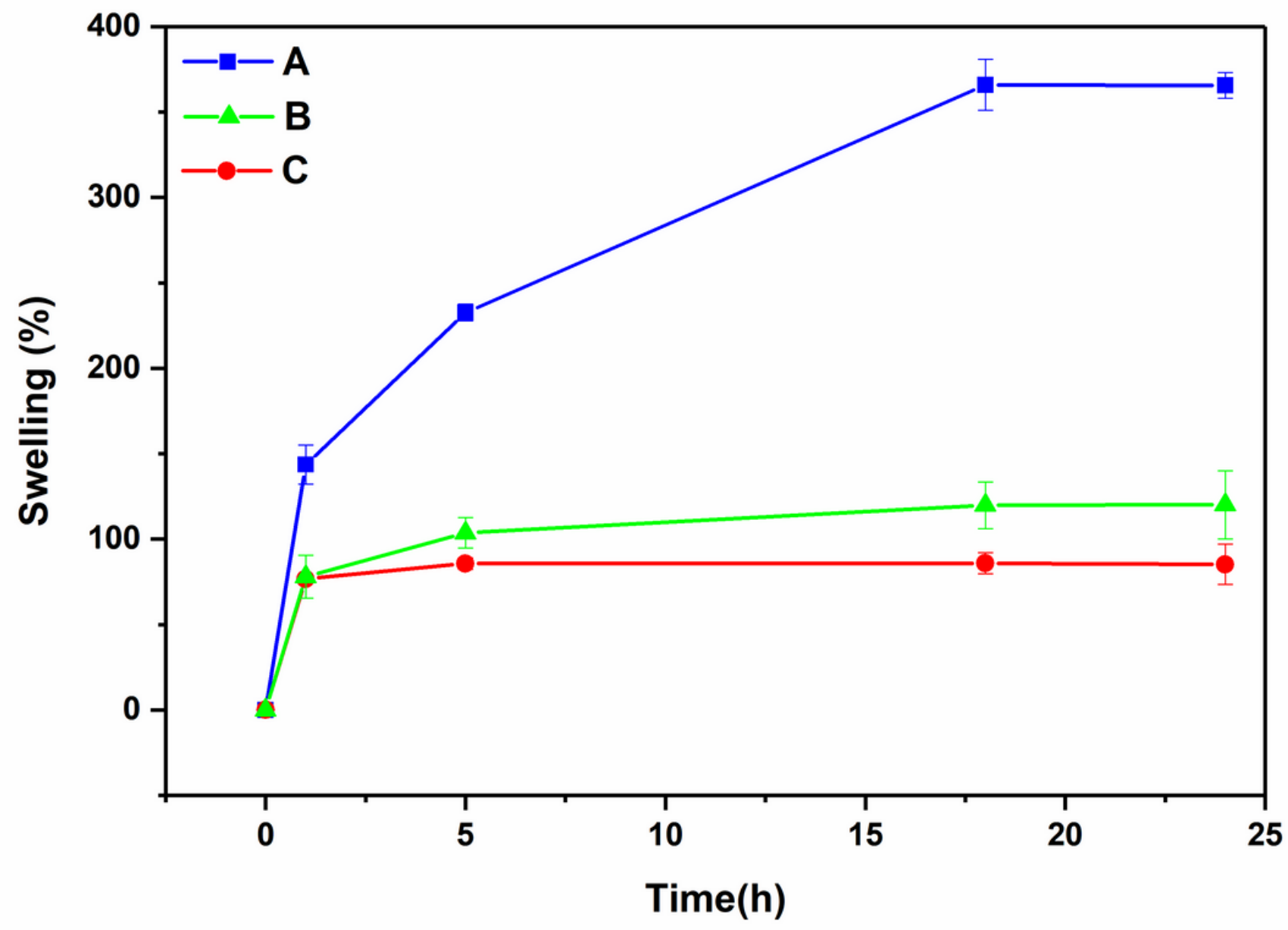

Figure 9

Swelling of GelMA hydrogels with different amount of cross-linker. Sample A $(0.005 \mathrm{~g})$, Sample B $(0.025 \mathrm{~g})$, Sample C $(0.05 \mathrm{~g})$. 


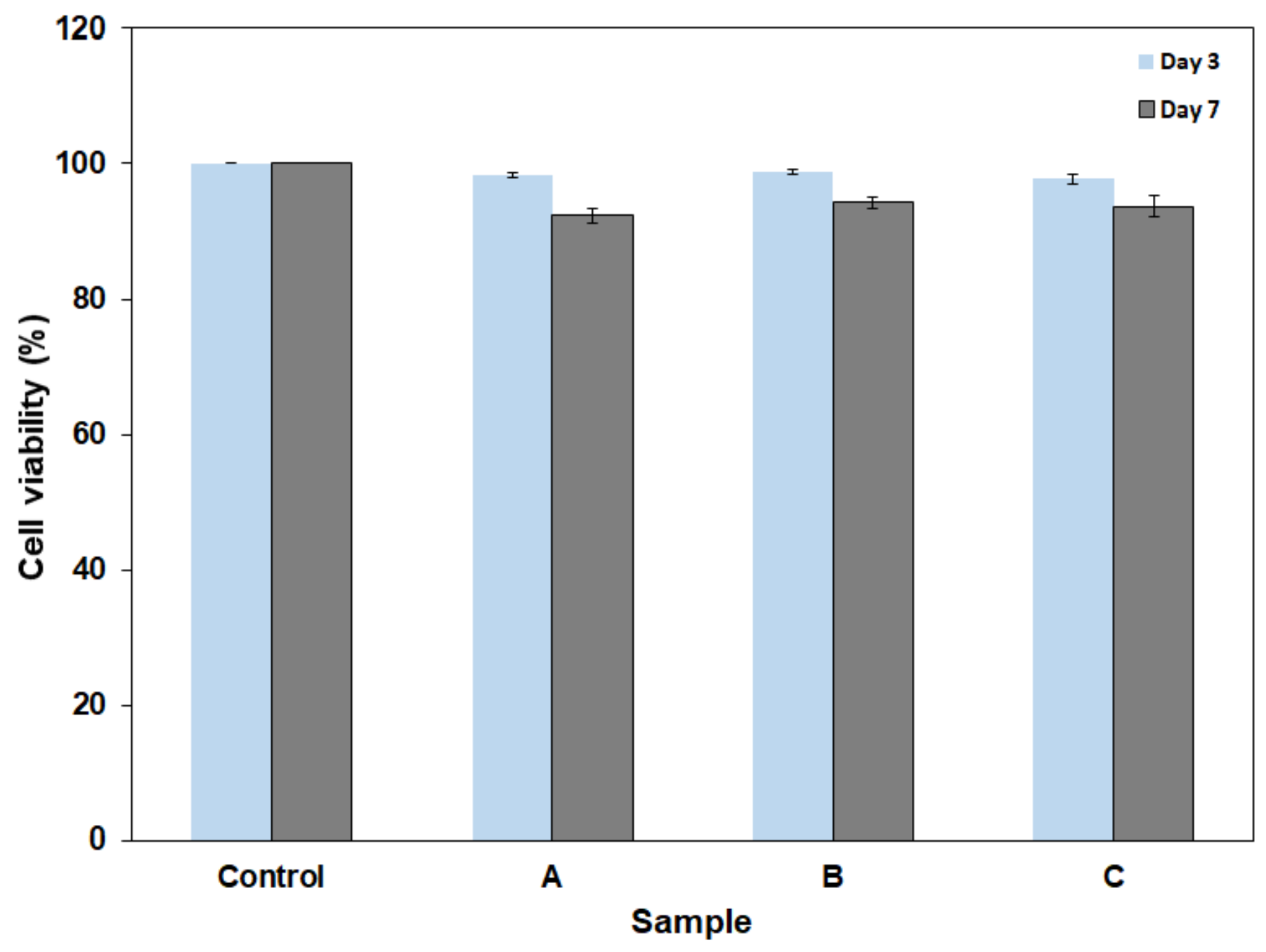

Figure 10

MTT assay of GelMA hydrogels with different amount of cross-linker. Sample A $(0.005 \mathrm{~g})$, Sample B $(0.025 \mathrm{~g})$, Sample C $(0.05 \mathrm{~g})$. 